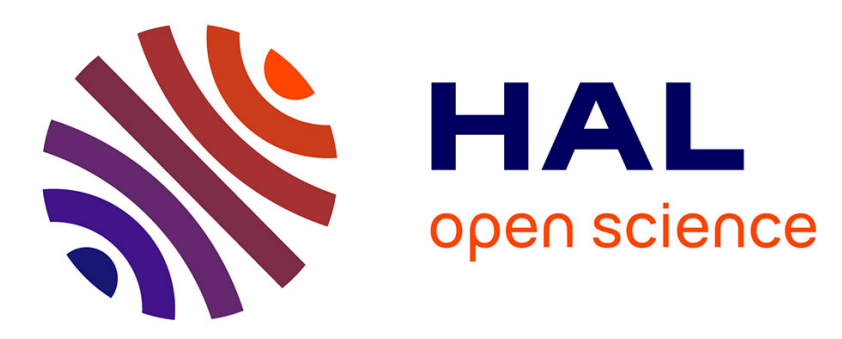

\title{
Questioning the causality of HTT CAG-repeat expansions in FTD/ALS
}

Quentin Thomas, Giulia Coarelli, Anna Heinzmann, Isabelle Le Ber, Maria del Mar Amador, Alexandra Durr

\section{- To cite this version:}

Quentin Thomas, Giulia Coarelli, Anna Heinzmann, Isabelle Le Ber, Maria del Mar Amador, et al. Questioning the causality of HTT CAG-repeat expansions in FTD/ALS: Response to Dewan et al., short HTT CAG-repeat expansions cause Huntington's disease with frontotemporal dementia-like phenotype.. Neuron, 2021, 109 (12), pp.1945-1946. 10.1016/j.neuron.2021.04.010 . hal-03500860

\section{HAL Id: hal-03500860 \\ https://hal.sorbonne-universite.fr/hal-03500860}

Submitted on 4 Jan 2022

HAL is a multi-disciplinary open access archive for the deposit and dissemination of scientific research documents, whether they are published or not. The documents may come from teaching and research institutions in France or abroad, or from public or private research centers.
L'archive ouverte pluridisciplinaire HAL, est destinée au dépôt et à la diffusion de documents scientifiques de niveau recherche, publiés ou non, émanant des établissements d'enseignement et de recherche français ou étrangers, des laboratoires publics ou privés. 


\section{Questioning the causality of HTT CAG-repeat expansions in FTD/ALS}

\section{You can't judge a frontal dementia by its cover}

Response to Dewan et al., short HTT CAG-repeat expansions cause Huntington's disease with frontotemporal dementia-like phenotype.

Dear Madam, Sir,

We read the article written by Dewan and collaborators entitled "Pathogenic Huntingtin Repeat Expansions in Patients with Frontotemporal Dementia and Amyotrophic Lateral Sclerosis" published in Neuron (Dewan et al., 2020) with great interest. In this work, the authors report whole-genome analyses in 4 different large cohorts of i) 2,442 patients diagnosed with frontotemporal dementia (FTD) and/or amyotrophic lateral sclerosis (ALS), ii) 2,599 patients diagnosed with Lewy body dementia (LBD), iii) 3,158 neurologically healthy individuals and iv) 3,674 individuals diagnosed with FTD/ALS from an additional independent "replication" cohort. They identified a total of 8 individuals carrying pathogenic HTT CAG-expansion repeats, 3 in the initial FTD/ALS cohort and 5 more in the independent "replication" cohort, but none in either the LBD cohort or among healthy controls. They showed that this overall $0.13 \%$ carrier rate $(8 / 6,116)$ is 4.4 times higher than observed among healthy individuals. Their anatomic pathology studies of some of the patients' brains show evidence of both polyglutamine and TDP-43 inclusions in cortical neurons, without signs of striatal degeneration. In light of this data, they conclude that their study broadens the phenotype resulting from $H T T$ repeat expansions and that HTT should be considered as a new causative gene of FTD/ALS. However, numerous elements in their manuscript raise questions as to the accuracy of this statement.

First, their claim that the carrier rate found in their cohort is 4.4 times higher than expected based on the $0.03 \%(10 / 31372)$ rate determined by Gardiner and collaborators and data from the 100000 genomes project. However, we previously described a $0.18 \%$ HTT CAG-expansion carrier rate in two independent cohorts of 2,838 and 1,255 subjects, including one of individuals with non-neurological disorders (Thion et al., 2016). This rate is higher to what Dewan and collaborators report, raising the concern that the cases they found are merely representative of the prevalence of the HTT expanded CAG repeats in the general 
population, which can be expected to be even higher in cohorts of patients with cognitive and motor symptoms.

Second, the scarce clinical descriptions of the cases do not seem, from a clinician's point of view, satisfactory enough to untangle the tricky differential diagnosis of HD versus FTD. The authors also do not seem to have taken into consideration the age-dependent penetrance of the pathological CAG-repeatexpansion in Huntington disease (HD) (Lee et al., 2012), nor its clinical heterogeneity. Indeed, late-onset HD patients do not always present with chorea but often, and for years, with isolated cognitive and/or behavioral changes (Paulsen et al., 2017). HD, like FTD, can give rise to altered social conduct, modified interpersonal relationships and deficits in executive functions. In their discussion, the authors point out the possibility that the patients "were simply misdiagnosed cases of atypical Huntington's disease", a statement we strongly agree with. Some of their patients did indeed display typical forms of HD. Detailed information about the clinical phenotype, neuropsychological and language evaluations, neuroimaging and pattern of brain atrophy would be of great interest. In fact, it seems that most patients could have had the clinical diagnosis of HD when analyzing their symptoms:

Patient \#1 (41 HTT CAG repeats) had tremor at age 68 and subsequently developed postural instability and oculomotor "problems" over a 3-year period, leading to the diagnosis of progressive supranuclear palsy. After a "very slowly progressive" disease course of 12 years, he became demented. While this 12-year evolution appears atypical for progressive supranuclear palsy, the clinical description, including the cognitive profile, are compatible with HD. Patient \#2 had behavioral changes at age 56 , with a familial history of dementia. Although the little available information, this description alone is highly congruent with HD. Patient \#3 had "language disturbances" at age 57 and was diagnosed with non-fluent primary progressive aphasia. Reduced verbal fluency is a frequent initial symptom of HD, and detailed information on the language evaluation, pattern of brain atrophy and clinical progression would be useful in determining whether she displayed other symptoms evocative of HD.

Regarding the three patients with ALS (patient \#5, \#7 and \#8), it is noteworthy that they all carried small CAG repeats, just above the pathological threshold (41 for patient \#5 and 40 for patients \#7 and \#8). As such, they may have died before displaying the full HD picture, given the wide range of age at onset, 
especially among smaller repeat expansions (Lee et al., 2012). Furthermore, their severely advanced clinical stage could have prevented physicians from noticing other neurological signs, a hypothesis that the authors mention in their discussion. Patient \#7 (40 CAG repeats) presented pyramidal weakness at age 76 and was subsequently diagnosed with FTD. More detailed clinical information would be interesting to the readers as the prior diagnosis of ALS may have mislead the diagnosis to FTD as it is a well-known association, while not considering HD.

The two remaining patients (patients \#4 and \#6) displayed clinical features that make HD an evident diagnosis, and both had family history evocative of HD (including one confirmed molecularly). Patient \#6 had behavioral changes typical of HD (irritability, apathy) and patient \#4's clinical description is on all fronts evocative of a typical form of juvenile HD, whose clinical presentation differs from adult-onset cases. Only $17 \%$ of juvenile HD patients display chorea at onset.

The authors point to anatomic pathology studies performed on some of the patients' brains revealing clear cortical predominance of the protein inclusions, and a mild or even complete lack of striatal atrophy as further evidence of the causality of the HTT CAG expansions in the genesis of FTD/ALS.

First, it is noteworthy that the 2 brains examined originated from the 2 patients with typical ALS phenotypes who died at a relatively young age (67 and 70 years old). Yet, previous anatomic pathology works have shown that striatal degeneration is correlated to the CAG repeat length. The fact that these patients had small CAG repeat expansions, that they either did not or barely displayed symptoms of HD, and that they had little striatal atrophy is therefore expected. Of note, the authors state that they found ubiquitinated nuclear inclusions in the striatum of both patients, even though the data is not shown.

Second, previous published works have shown that there is no correlation between the CAG repeat expansion and cortical neuronal loss, and that cortical neuron loss is often more severe than what would be expected from the CAG repeat length and duration of the disease (Nana et al., 2014). This finding is consistent with the authors' findings which describe significant cortical neuron loss as compared to striatal degeneration. Their statement that this finding is dissuasive of a diagnosis of HD is however incorrect. Cortical neuron loss often correlates with clinical presentation, and the fact that the authors find mainly huntingtin 
inclusions in the prefrontal cortex is in accordance with the patients' phenotypes which were mostly of cognitive nature (Nana et al., 2014).

Third, the authors describe extensive spinal cord and motor neuron loss. This finding is expected not only in ALS anatomic pathology findings, but in HD as well which has also been found to generate motor neuron loss (Phillips et al., 2015). The co-existence of the 2 disorders may thus generate an even greater loss of motor neurons.

Fourth, the coexistence of huntingtin and TDP43 aggregates in brains of HD patients has already been reported and it has been shown in human cell cultures that mutant huntingtin leads to phosphorylation and aggregation of TDP43 (Coudert et al., 2019).

With regard to all of the previously discussed elements, Dewan and collaborators' work, while not proving that HTT is a new gene of FTD/ALS, is a perfect illustration of how tricky clinical diagnosis of HD can be. It also highlights that HD is still synonymous with choreic movements, for at least part of the medical community, and that there is still much work to do in order to raise awareness of the numerous shapes that the disease can take. The case of patient \#4 is particularly illustrative of this, as her clinical description is typical of a juvenile form of HD and should have led to its clinical diagnosis. In a previous work we showed that FTD/ALS and HD may clinically overlap by identifying a pathogenic VCP missense variant in a patient diagnosed with HD. It is thus not surprising to see that diagnoses of ALS and FTD are made in patients with HD. The fact that authors did not find expanded HTT CAG repeat carriers among the LBD group is also logical as the cognitive phenotype differs drastically between HD and LBD.

In the discussion, the authors highlight the importance of an accurate molecular diagnosis to provide adequate genetic counselling, and we fully agree with them on this point. Although the rapid improvement of sequencing technologies has led to an incredible increase in medical knowledge, results generated by such tools should always be interpreted with great caution and in the perspective of current medical knowledge. 
Quentin Thomas ${ }^{1}$, MD, Giulia Coarelli ${ }^{1,2}$, MD, Anna Heinzmann ${ }^{1,2}$, MD, Isabelle Le Ber ${ }^{2,3}, \mathrm{MD}$ PhD, Maria del Mar Amador ${ }^{1,4}, \mathrm{MD}$, Alexandra Durr ${ }^{1,2}, \mathrm{MD}$ PhD

1 AP-HP, Genetic Department, University Hospital Pitié-Salpêtrière, Paris, France Sorbonne 2 Sorbonne University, Paris Brain Institute, APHP, INSERM, CNRS, Paris, France

3 AP-HP, National Reference center "rare and young dementias", IM2A, University Hospital PitiéSalpêtrière, Paris, France

4 Sorbonne University, Department of Neurology, National Reference center ALS Paris, Assistance Publique Hôpitaux de Paris, University Hospital Pitié-Salpêtrière, Paris, France

\section{Corresponding author and lead contact}

Pr Alexandra Durr

Paris Brain Institute (Institut du Cerveau-ICM)

47 boulevard de l'Hôpital

75651 Paris CEDEX 13

France

alexandra.durr@icm-institute.org

\section{Acknowledgement}

No funding was received for the writing of this article.

\section{Competing Interests}

The authors declare no competing interests. 


\section{References}

Coudert, L., Nonaka, T., Bernard, E., Hasegawa, M., Schaeffer, L., and Leblanc, P. (2019). Phosphorylated and aggregated TDP-43 with seeding properties are induced upon mutant Huntingtin ( $\mathrm{mHtt}$ ) polyglutamine expression in human cellular models. Cell. Mol. Life Sci. 76.

Dewan, R., Chia, R., Ding, J., Hickman, R.A., Stein, T.D., Abramzon, Y., Ahmed, S., Sabir, M.S., Portley, M.K., Tucci, A., et al. (2020). Pathogenic Huntingtin Repeat Expansions in Patients with Frontotemporal Dementia and Amyotrophic Lateral Sclerosis. Neuron 1-13.

Lee, J.M., Ramos, E.M., Lee, J.H., Gillis, T., Mysore, J.S., Hayden, M.R., Warby, S.C., Morrison, P., Nance, M., Ross, C.A., et al. (2012). CAG repeat expansion in Huntington disease determines age at onset in a fully dominant fashion. Neurology 78, 690-695.

Nana, A.L., Kim, E.H., Thu, D.C.V., Oorschot, D.E., Tippett, L.J., Hogg, V.M., Synek, B.J., Roxburgh, R., Waldvogel, H.J., and Faull, R.L.M. (2014). Widespread heterogeneous neuronal loss across the cerebral cortex in Huntington's disease. J. Huntingtons. Dis. 3, 45-64.

Paulsen, J.S., Miller, A.C., Hayes, T., and Shaw, E. (2017). Cognitive and behavioral changes in Huntington disease before diagnosis. In Handbook of Clinical Neurology, (Elsevier B.V.), pp. 69-91.

Phillips, O., Squitieri, F., Sanchez-Castaneda, C., Elifani, F., Griguoli, A., Maglione, V., Caltagirone, C., Sabatini, U., and Di Paola, M. (2015). The corticospinal tract in huntington's disease. Cereb. Cortex 25, 2670-2682.

Thion, M.S., Tézenas Du Montcel, S., Golmard, J.L., Vacher, S., Barjhoux, L., Sornin, V., Cazeneuve, C., Bièche, I., Sinilnikova, O., Stoppa-Lyonnet, D., et al. (2016). CAG repeat size in Huntingtin alleles is associated with cancer prognosis. Eur. J. Hum. Genet. 24, 1310-1315. 